\title{
Direct binding of RNF8 to SUMO2/3 promotes cell survival following DNA damage
}

\author{
LILI LIANG ${ }^{1}$, ZHENZHU ZHANG $^{2},{\text { JIANDONG } \text { LI }^{2}, \text { JIAOXIANG WU }}^{2}$, \\ LIKUI WANG ${ }^{2}$, WENLIN HUANG ${ }^{2,3}$ and SHIJUAN GAO ${ }^{2,4}$
}

\begin{abstract}
${ }^{1}$ Division of Digestive Endoscopy, The First Affiliated Hospital of Dalian Medical University, Dalian, Liaoning 116011; ${ }^{2}$ CAS Key Laboratory of Pathogenic Microbiology and Immunology, Institute of Microbiology, Chinese Academy of Sciences, Beijing 100101; ${ }^{3}$ Sun Yat-Sen University Cancer Center, State Key Laboratory of Oncology in South China, Collaborative Innovation Center of Cancer Medicine, Guangzhou, Guangdong 510060; ${ }^{4}$ The Key Laboratory of Remodeling-Related Cardiovascular Diseases, Collaborative Innovation Center for Cardiovascular Disorders, Beijing Institute of Heart, Lung and Blood Vessel Diseases; Beijing Anzhen Hospital, Capital Medical University, Beijing 100029, P.R. China
\end{abstract}

Received March 9, 2016; Accepted March 13, 2017

DOI: $10.3892 / \mathrm{mmr} .2017 .7624$

\begin{abstract}
Ring finger protein 8 (RNF8), an FHA/RING domain containing E3 ubiquitin ligase, is critical in supporting genome integrity by facilitating the assembly of multiple DNA repair proteins at DNA lesions following DNA damage. In the present study, a search for novel binding partners of RNF8 was performed using a yeast two-hybrid screening assay, and small ubiquitin-like modifier (SUMO)2/3 was identified as one of the major RNF8-binding candidates. GST pull-down and immunoprecipitation assays revealed that RNF8 bound directly and noncovalently to SUMO2/3, but not to SUMO1, and that the FHA domain of RNF8 was required for the binding to SUMO2/3. Furthermore, RNF8 co-localized with SUMO2/3 at sites of DNA lesions in response to ionizing radiation, as revealed by immunofluorescence assay. Survival assay indicated that the depletion of RNF8 and SUMO2/3 resulted in decreased cellular resistance to genotoxic stress. These data suggested that the binding of RNF8 to SUMO2/3 promoted the response to DNA damage.
\end{abstract}

\section{Introduction}

DNA damage occurs constantly due to intrinsic DNA metabolic activities and extrinsic agents, including irradiation and various DNA-damaging chemicals. A double-strand break (DSB) is one of the most serious lesions as it can

Correspondence to: Dr Lili Liang, Division of Digestive Endoscopy, The First Affiliated Hospital of Dalian Medical University, 222 Zhong Shan Road, Dalian, Liaoning 116011, P.R. China

E-mail: 1ily-7613@163.com

Key words: ring finger protein 8, small ubiquitin-like modifier, DNA damage response, protein interaction, cell survival lead to genome rearrangements if not properly sensed and repaired (1). To respond to this challenge, cells elicit a coordinated DNA-damage response, which includes DNA repair, checkpoint activation, and consequent cell cycle arrest and/or apoptosis to maintain genomic integrity $(2,3)$.

The involvement of ring finger protein 8 (RNF8), an FHA domain and RING finger motif-containing protein, is important in the cellular response to DNA damage. RNF8 is rapidly accumulated at DSBs via the interaction of its $\mathrm{N}$-terminal FHA domain with the phosphorylated mediator of DNA-damage checkpoint 1 (MDC1), which binds to the phosphorylated $\mathrm{H} 2 \mathrm{AX}(\gamma-\mathrm{H} 2 \mathrm{AX})$ accumulating at the DNA lesions, where it functions as an E3 ligase to ubiquitinate $\mathrm{H} 2 \mathrm{~A}$, $\mathrm{H} 2 \mathrm{AX}$ and other unknown proteins (4-8). The ubiquitination of $\mathrm{H} 2 \mathrm{~A}$ and $\mathrm{H} 2 \mathrm{AX}$ recruits RNF168, another E3 ligase, to sites of DSBs to amplify the RNF8-dependent H2A ubiquitination and promote the formation of Lys63-linked ubiquitin chains for the recruitment of p53-binding protein 1 (53BP1), breast cancer 1 (BRCA1) and other damage repair proteins (9). In this manner, RNF8 serves as a molecular link between the protein phosphorylation and protein ubiquitination pathways, which are essential in the DNA damage response.

Small ubiquitin-like modifier (SUMO) modification is another post-translational modification known to regulate the DNA damage response. In mammals, three SUMO paralogues, SUMO1, SUMO2 and SUMO3, are expressed. SUMO2 and SUMO3 differ by three N-terminal residues, whereas SUMO1 is $45 \%$ identical to either SUMO2 or SUMO3. All three SUMO species can be covalently attached to target proteins by forming an isopeptide bond between the target lysine and the activated SUMO c-terminal glycine residue by a mechanism similar to that of ubiquitination. SUMO modification has been shown to be required for complete accumulation of DNA damage-repair proteins, including RNF8, RNF168, BRCA1 and 53BP1, at sites of DNA lesions (10). Furthermore, sumoylation of DSB response-associated ubiquitin ligases, for example BRCA1, enhances ubiquitin ligase activity (11). 
In addition to modification by SUMO, target proteins can bind noncovalently to SUMO via its short hydrophobic region, known as SUMO interaction motif (SIM), to alter its cellular localization and/or activity (12-14). In the majority of cases, however, SUMO-mediated protein-protein interactions during the DNA damage response and the consequent effect on genomic integrity remain to be elucidated.

In the present study, a yeast two-hybrid assay was performed to identify RNF8-binding proteins, which identified SUMO2/3 as its binding partner. The data presented showed that RNF8 bound directly and noncovalently to SUMO2/3, but not to SUMO1. The accumulation of RNF8 and SUMO2/3 at sites of DNA lesions contributed to DSB repair following ionizing radiation.

\section{Materials and methods}

Plasmids. The full-length human RNF8 gene was obtained by polymerase chain reaction (PCR) from the $\mathrm{p}-\mathrm{RNF} 8$ plasmid (provided by Professor Tiebang Kang of Sun Yat-sen University Cancer Center, Guangzhou, China) and subcloned into the pBridge vector (Clontech Laboratories, Inc., Mountainview, CA, USA) carrying the Gal4 DNA-binding domain, pcDNA3.0-Flag vector and pEGFP-N1 vector (Clontech Laboratories, Inc.). Human SUMO1, SUMO2 and SUMO3 were PCR-amplified from the HeLa cDNA library (Clontech Laboratories, Inc.) and cloned into the pGEX4T-1, pDsRed2-N1, pGAD-T7 or pXJ40-HA vector. The SUMO3 $\left(\mathrm{G}_{91} \mathrm{G}_{92} \rightarrow \mathrm{A}_{91} \mathrm{~A}_{92}\right)$ mutation was generated by PCR with mutation-specific primers and cloned into the $\mathrm{pXJ} 40$-HA vector (provided by Professor Xuemin Zhang of National Center of Biomedical Analysis, Beijing, China). The primer sequences used for PCR amplification were as follows: SUMO1 forward, 5'-ATGTCTGACCAGGAGGCAAAACCTTC-3 and reverse, 5'-CTA AACTGT TGA ATGACCCCCCGTT-3; SUMO2 forward, 5'-ATGGCCGACGAAAAGCCCAAG-3 and reverse, 5'-TCAGTAGACACCTCCCGTCTGCTG-3; SUMO3 forward, 5'-ATGTCCGAGGAGAAGCCCAAG-3 and reverse, 5'-CTAGAAACTGTGCCCTGCCAG-3'. The cycle conditions included an initial denaturation step at $94^{\circ} \mathrm{C}$ for $5 \mathrm{~min}$ followed by 35 cycles of $94^{\circ} \mathrm{C}$ for $30 \mathrm{sec}, 58^{\circ} \mathrm{C}$ for $30 \mathrm{sec}$ and $72^{\circ} \mathrm{C}$ for $1 \mathrm{~min}$ and then a final extension at $72^{\circ} \mathrm{C}$ for $5 \mathrm{~min}$.

Antibodies and reagents. The mouse anti-Flag antibody (cat. no. F3165) was purchased from Sigma-Aldrich; Merck Millipore (Darmstadt, Germany). Mouse anti-hemagglutinin (HA; cat. no. sc-7392) antibody was provided by Santa Cruz Biotechnology, Inc. (Santa Cruz, CA, USA) and goat anti-HA antibody was from GenScript (Nanjing, China). Rabbit anti-phospho-H2A.X (cat.no. 9718) was from Cell Signaling Technology, Inc. (Danvers, MA, USA). Fluorescein isothiocyanate (FITC)-conjugated anti-mouse IgG (cat. no. 715-095-151) and tetramethyl rhodamine isocyanate (TRITC)-conjugated anti-goat IgG (cat. no. 305-025-003) were from Jackson ImmunoResearch Laboratories, Inc. (West Grove, PA, USA). Glutathione-Sepharose 4B beads were purchased from GE Healthcare Life Sciences (Uppsala, Sweden) and Protein G beads were from Santa Cruz Biotechnology, Inc. (Santa Cruz, CA, USA). The small interfering (si)RNA oligonucleotides against RNF8, SUMO1 and SUMO2/3 were chemically synthesized by Guangzhou RiboBio Co., Ltd. (Guangzhou, China). siRNA against RNF8 was 5'AGAAUGAGCUCCAAU GUAUU3'; siRNA against SUMO1 was 5'GGACAGGAU AGCAGUGAGA3'; and siRNA against SUMO2/3 was 5'CAA UGAGGCAGAUCAGAUU3'.

Yeast two-hybrid screening. Full length cDNA from human RNF8 was cloned in frame with the Gal4 DNA-binding domain in pBridge. pBD-RNF8 was co-transfected into the Saccharomyces cerevisiae strain CG1945 together with a human fetal brain cDNA library (Clontech Laboratories, Inc., CA, USA) using the lithium acetate. Transformants $\left(\sim 10^{7}\right)$ were selected on 100-mm Sabouraud Dextrose/-Trp-Leu-His dropout plates $\left(30^{\circ} \mathrm{C} ; 4-5\right.$ days) for the primary screening and were analyzed using a LacZ assay for the second screening. Following rescue, the potential positive plasmids were isolated and re-transformed into the CG1945 yeast strain containing the RNF8 bait plasmid. Only clones, which were confirmed to be positive by at least two independent tests were selected for specific interactions and sequenced.

Cell culture and transfection. HeLa human epithelial carcinoma cells and U2OS human osteosarcoma cells were obtained from Peking Union Medical College (Beijing, China) and grown in DMEM supplemented with $8 \%$ fetal bovine serum (Gibco; Thermo Fisher Scientific, Inc., Waltham, MA, USA), $100 \mathrm{U} / \mathrm{ml}$ penicillin and $100 \mu / \mathrm{ml}$ streptomycin, at $37^{\circ} \mathrm{C}$ in a humidified atmosphere containing $5 \% \mathrm{CO}_{2}$ and $95 \%$ air. Cell transfection was performed using Lipofectamine 2000 (Invitrogen; Thermo Fisher Scientific, Inc.) according to the manufacturer's protocol.

Ionizing radiation. The $\mathrm{U} 2 \mathrm{OS}$ cells were plated into a 6 -well plate at a density of $6 \times 10^{5}$ cells/well and irradiated using a Biological X-ray irradiator (RS 2000; Rad Source Technologies, Inc., Suwanee, GA, USA) at $160 \mathrm{kV}$ and $25 \mathrm{~mA}$, with a dose of $10 \mathrm{~Gy}$ and a dose rate of $2 \mathrm{~Gy} / \mathrm{min}$.

Western blot analysis. Total proteins were extracted using cell lysis buffer (Cell Signaling Technology,Inc.). Whole cell lysates were quantified using a BCA Protein assay kit (Beyotime Institute of Biotechnology, Shanghai, China) and $20 \mu \mathrm{g}$ of protein was loaded per lane to separate on 10 or $12 \%$ sodium dodecyl sulfate polyacrylamide gels. Subsequently, the separated proteins were transferred onto polyvinylidene difluoride membranes. The membrane was then blocked with 5\% non-fat milk in Tris-buffered saline (TBS) for $2 \mathrm{~h}$ at room temperature and incubated overnight at $4^{\circ} \mathrm{C}$ anti-Flag $(1: 1,000)$ and anti-HA $(1: 1,000)$ antibodies. After washing three times for 10 min each with TBST, the membrane was incubated for $2 \mathrm{~h}$ at room temperature with a horseradish peroxidase-conjugated secondary antibody $(1: 5,000)$. The bands were visualized using an ECL detection kit (Applygen Technologies, Inc., Beijing, China).

Glutathione S-transferase (GST) pull-down assay. Recombinant GST and GST-fused human SUMO2 or SUMO3 proteins were purified from Escherichia coli BL21 (DE3) (New England BioLabs, Inc., Ipswich, MA, USA) cells using glutathione-sepharose $4 \mathrm{~B}$ beads. The cell lysates 
from the HeLa cells transiently transfected with Flag-RNF8, Flag-RNF8 ( $\triangle$ FHA) and Flag-RNF8 ( $\triangle$ RING) were incubated with purified GST, GST-SUMO2 and GST-SUMO3 at $4{ }^{\circ} \mathrm{C}$ overnight. The beads were then washed four times with lysis buffer. The bound proteins were eluted by boiling in 2X SDS loading buffer and analyzed using western blot analysis with the anti-Flag antibody.

Immunoprecipitation. The HeLa cells were seeded into $10 \mathrm{~cm}$ diameter dishes $\left(1 \times 10^{6}\right.$ cells/dish) and co-transfected with Flag-RNF8 and HA-SUMO1, HA-SUMO2, HA-SUMO3 or HA-SUMO3 $\mathrm{M}\left(\mathrm{G}_{91} \mathrm{G}_{92} \rightarrow \mathrm{A}_{91} \mathrm{~A}_{92}\right)$. At $36 \mathrm{~h}$ post-transfection, the cells were harvested and lysed using cell lysis buffer (Cell Signaling Technology, Inc.). The cell lysates were centrifuged at $12,000 \mathrm{x} \mathrm{g}$ for $10 \mathrm{~min}$ at $4^{\circ} \mathrm{C}$ and the supernatants were incubated with anti-HA antibody at $4^{\circ} \mathrm{C}$ for $2 \mathrm{~h}$. Protein $\mathrm{G}$ agarose beads were then added, and the mixture was incubated at $4^{\circ} \mathrm{C}$ overnight. The beads were washed three times with cell lysis buffer, boiled in 2X SDS loading buffer for $6 \mathrm{~min}$, and the precipitated proteins were subjected to western blot analysis.

Immunostaining procedure. To observe the cellular co-localization of RNF8 and SUMO2/3, the U2OS cells were co-transfected with RNF8-GFP and SUMO2-Red or SUMO3-Red for $28 \mathrm{~h}$. The cells were then washed with PBS and incubated with $4 \%$ paraformaldehyde for $20 \mathrm{~min}$ at room temperature. Following washing with PBS, the coverslips were mounted onto glass slides and observed under a Leica confocal microscope (Leica Microsystems GmbH, Wetzlar, Germany).

To detect whether RNF8 and SUMO2/3 accumulated at DNA-damage sites, the U2OS cells were seeded at a density of $6 \times 10^{5}$ cells/well on cover slips in a 6 -well plated and transfected with Flag-RNF8 and HA-SUMO2 for $28 \mathrm{~h}$. The cells were irradiated with a $10 \mathrm{~Gy}$ dose, followed by recovery for $6 \mathrm{~h}$. The cells were then washed three times with PBS, fixed with $4 \%$ paraformaldehyde and permeabilized with $0.2 \%$ Triton X-100 for $4 \mathrm{~min}$ at room temperature. The samples were blocked with 5\% goat serum (cat. no. 5425, Cell Signaling Technology, Inc.) followed by incubation with anti-Flag, anti-HA and anti-phospho- $\mathrm{H} 2 \mathrm{AX}$ antibodies at $37^{\circ} \mathrm{C}$ for $45 \mathrm{~min}$. Following incubation with TRITC- and FITC-conjugated secondary antibodies, the cells were visualized under a Leica confocal microscope.

Survival assay. The U2OS cells were seeded in 12-well plate at a density of $2 \times 10^{5}$ cells/well and transfected with either control siRNA or siRNA against RNF8, SUMO1 or SUMO2/3 using Lipofectamine 2000 reagent according to the manufacturer's instructions. At $48 \mathrm{~h}$ post-transfection, the cells were exposed to different dose of radiation (1-3 Gy) and left to grow for 10 days. The cells were then fixed and stained with crystal violet, and the numbers of colonies were counted using a light microscope. The experiment was performed three times.

Statistical analysis. The samples were assayed in triplicate. Data are presented as the mean \pm standard deviation. The results shown are representative of at least three experiments. Differences were determined using one-way analysis of variance followed by Student's t-test. $\mathrm{P}<0.05$ was considered to indicate a statistically significant difference.

\section{Results}

RNF8 binds directly and noncovalently to SUMO2/3. To better understand the roles of RNF8 in DNA damage signaling, the present study performed yeast-two hybrid assays to identify RNF8-binding proteins. Among the proteins recognized by RNF8, SUMO2/3 was of particular interested (Fig. 1A and B). The interaction between RNF8 and SUMO2/3 was further confirmed in the in vitro GST-pull down assay (Fig. 1C). Mammalian cells express three paralogs: SUMO2 and SUMO3, which are $96 \%$ identical, and SUMO1, which is $45 \%$ identical to SUMO2/3. Therefore, the present study investigated whether RNF8 binds to SUMO1. Using an in vivo co-immunoprecipitation assay in HeLa cells overexpressing Flag-tagged RNF8 and HA-tagged SUMO1, SUMO2 or SUMO3, it was found that RNF8 bound efficiently to SUMO2 and SUMO3, but almost no binding was observed to SUMO1 (Fig. 1D). It is known that SUMO can interact covalently and/or noncovalently with target proteins. To distinguish whether the association of RNF8 with SUMO2/3 was covalent or noncovalent, the present study constructed a nonconjugatable form of SUMO3 $\left(\mathrm{G}_{91} \mathrm{G}_{92} \rightarrow \mathrm{A}_{91} \mathrm{~A}_{92}\right)$ and contransfected it with Flag-RNF8 into HeLa cells. As shown in Fig. 1D, mutation of SUMO3 did not affect binding to RNF8, indicating that RNF8 binds noncovalently to SUMO3. Finally, a Leica confocal microscope was used to detect the cellular co-localization of RNF8 with SUMO2/3 in HeLa cells. As shown in Fig. 1E, SUMO2-Red and SUMO3-Red co-localized specifically with RNF8-GFP in the nucleus, but not with GFP. Together, these data suggested that RNF8 was able to bind directly and noncovalently to SUMO2/3.

Mapping the SUMO2/3 binding domain in RNF8. In order to map the SUMO2/3 binding domain in RNF8 in the present study, a series of RNF8 truncation mutants were generated (Fig. 2A). The results from one-to-one yeast two-hybrid assays performed with RNF8 deletions and SUMO2 indicated that the region between amino acids 17 and 120 of RNF8, which includes the FHA domain, is important for binding to SUMO2 (Fig. 2B). This result was further confirmed in the in vitro GST-pull down assay. As shown in Fig. 2C, deletion of the RING domain bound SUMO2 and wild-type RNF8, whereas truncation of the FHA domain did not bind to GST-SUMO2. Thus, deletion analysis revealed that the RNF8 FHA domain is required for binding to SUMO2/3. The FHA domain-mediated interaction between RNF8 and SUMO2/3 was also confirmed using a co-immunoprecipitation assay with FHA domain deletion mutants of RNF8. As shown in Fig. 2D, the FHA domain was found to bind efficiently to SUMO2. Furthermore, SUMO2 co-localized specifically with RNF8 in the nucleus, but not with the RNF8 FHA deletion mutant (Fig. 2E).

Interaction of RNF8 with SUMO2/3 increases cellular resistance to genotoxic stress. Upon DNA damage, checkpoint proteins localize to chromatin structures at the vicinity of DNA breaks. Thus, focus formation following DNA damage can be observed using immunostaining. RNF8 is well known to accumulate at the chromatin following DNA damage. The present study also observed RNF8 focus formation following ionizing radiation and theses foci co-localized with the DNA damage 

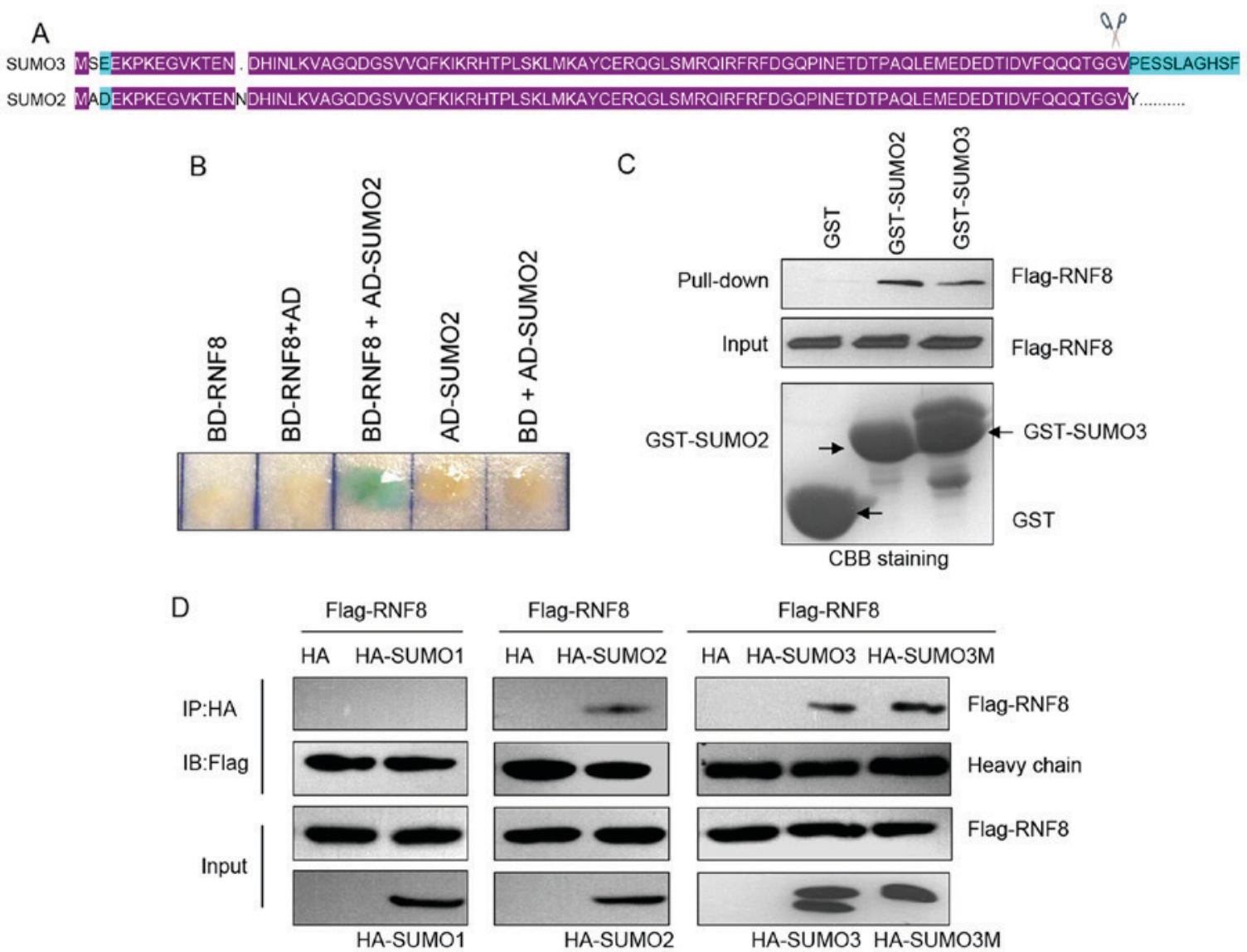

$\mathrm{E}$
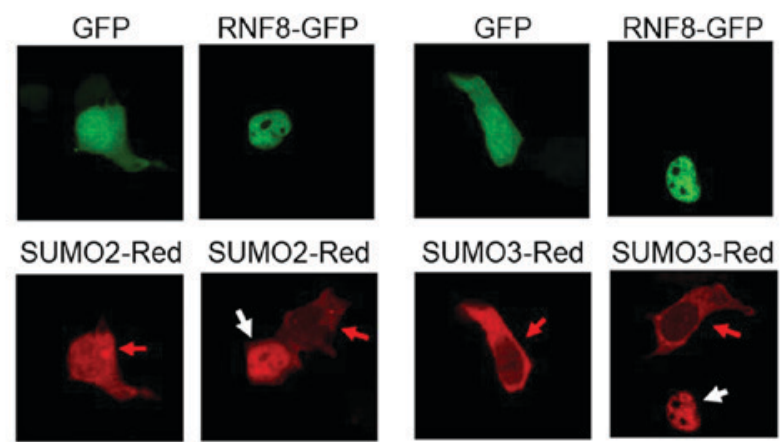

SUMO2-Red

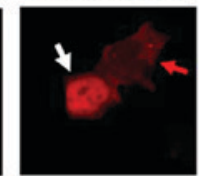

SUMO3-Red

SUMO3-Red
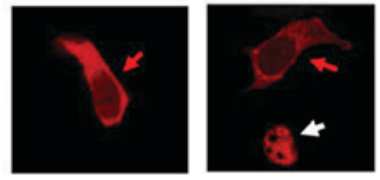

Overlay

Overlay
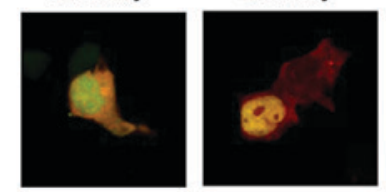

Overlay

Overlay
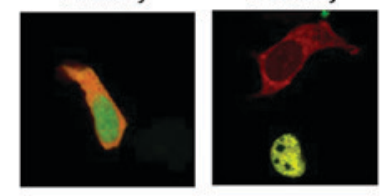

Figure 1. RNF8 binds to SUMO2/3 noncovalently. (A) Alignment of SUMO2 and SUMO3. SUMO2 and SUMO3 were 96\% identical. (B) Yeast two-hybrid assay analysis of the interaction between RNF8 and SUMO2. A blue product indicated a positive interaction. (C) GST pull-down assay showing the direct interaction between RNF8 and SUMO2/3. (D) Co-immunoprecipitation of Flag-RNF8 and HA-SUMO2/3 in HeLa cells. HeLa cells were transfected with plasmids encoding Flag-tagged RNF8 with or without plasmids encoding HA-SUMO1, HA-SUMO2, HA-SUMO3 and its mutation $\left(\mathrm{G}_{91} \mathrm{G}_{92} \rightarrow \mathrm{A}_{91} \mathrm{~A}_{92}\right)$. Immunoprecipitation and immunoblotting were performed using antibodies, as indicated. (E) Co-localization of RNF8 and SUMO2/3 in HeLa cells. Plasmids encoding RNF8-GFP and SUMO2-Red or SUMO3-Red were transiently transfected into HeLa cells for $28 \mathrm{~h}$. The cells were fixed and observed under a Leica confocal microscope. Magnification, x630. White arrows indicate RNF8-GFP-expressing cells. Red arrows indicate RNF8-GFP-non-expressing cells. GST, glutathione S-transferase; RNF8, ring finger protein 8; SUMO, small ubiquitin-like modifier; CBB, coomassie brilliant blue; HA, hemagglutinin.

marker, $\gamma$-H2AX. Furthermore, SUMO2 foci were readily visualized following DNA damage and the SUMO2 foci overlapped with those of RNF8 (Fig. 3A). Thus, it appeared that the binding of RNF8 to SUMO2/3 was involved in the RNF8 response to genotoxic stress. Therefore, the present study examined whether the binding of RNF8 to SUMO2/3 was required for cell survival following DNA damage. As shown in Fig. 3B, consistent with the expected results, the transient knockdown of RNF8, SUMO1 or SUMO2/3 resulted in a significant increase in cellular radiation sensitivity. Of note, it was found that the knockdown of RNF8 together with SUMO2/3 had an additive effect on increased cellular radiation sensitivity, 
A

B
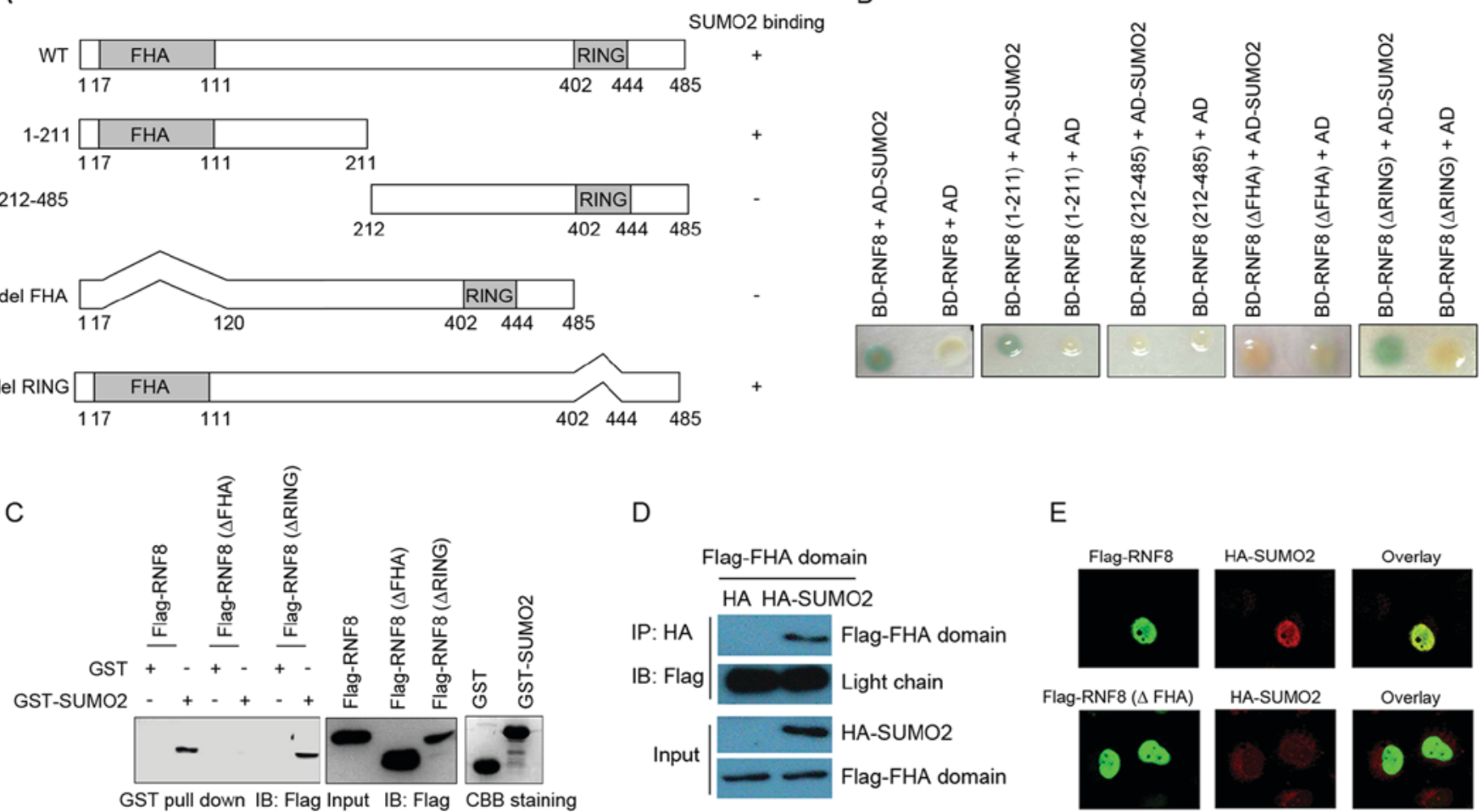

$E$

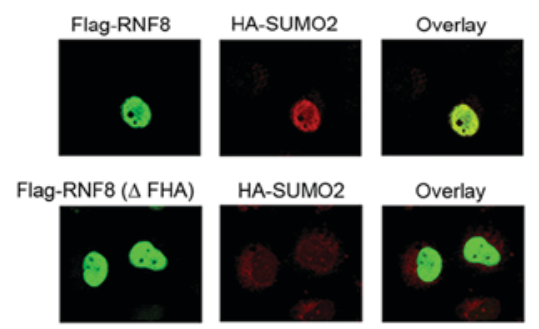

Figure 2. Mapping the SUMO2/3 interaction domain in RNF8. (A) Schematic representation of the deletion mutants of RNF8. The binding of SUMO2 by RNF8 deletion mutants is indicated on the right based on the one-to-one yeast two-hybrid assays. (B) Yeast strain CG1945 was co-transformed with the bait and prey plasmids as indicated. A single yeast colony was subjected to a liquid $\beta$-gal assay, with the presence of a blue product indicating a positive interaction. (C) FHA domain of RNF8 was required for binding to SUMO2 based on the in vitro GST pull-down assay. Lysates of HeLa cells transfected with Flag-RNF8, Flag-RNF8 ( $\triangle \mathrm{FHA})$ and Flag-RNF8 ( $\triangle \mathrm{RING)}$ were incubated with GST or GST-SUMO2 at $4^{\circ} \mathrm{C}$ overnight. The bound proteins were analyzed using western blot analysis with an anti-Flag antibody. (D) Co-immunoprecipitation showing the interaction of HA-SUMO2 and Flag-FHA domain of RNF8 in HeLa cells (E) Immunofluorescence assay showing nuclear co-localization of SUMO2 and WT RNF8, but not with the RNF8 FHA deletion mutant. The cells were observed under a Leica confocal microscope. Magnification, x630. GST, glutathione S-transferase; RNF8, ring finger protein 8; SUMO, small ubiquitin-like modifier; CBB, coomassie brilliant blue; HA, hemaglutinin; WT, wild-type.

A

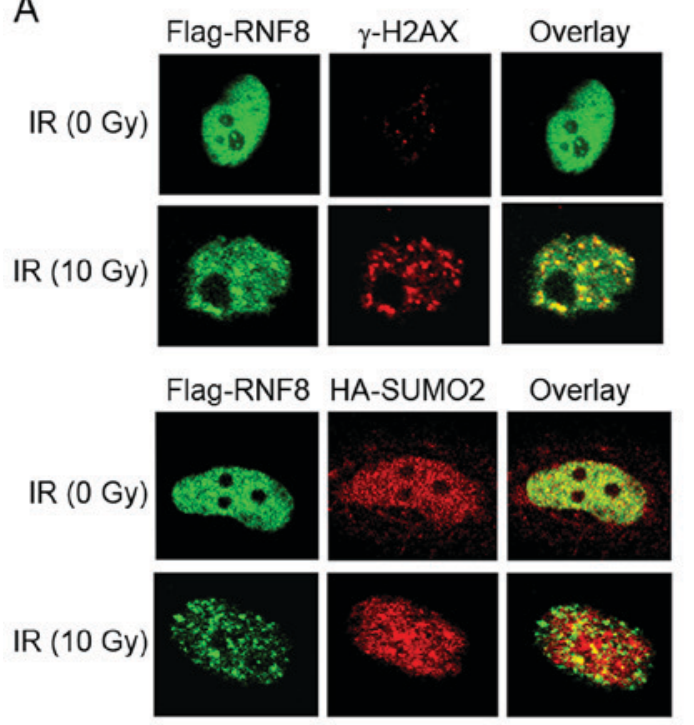

B

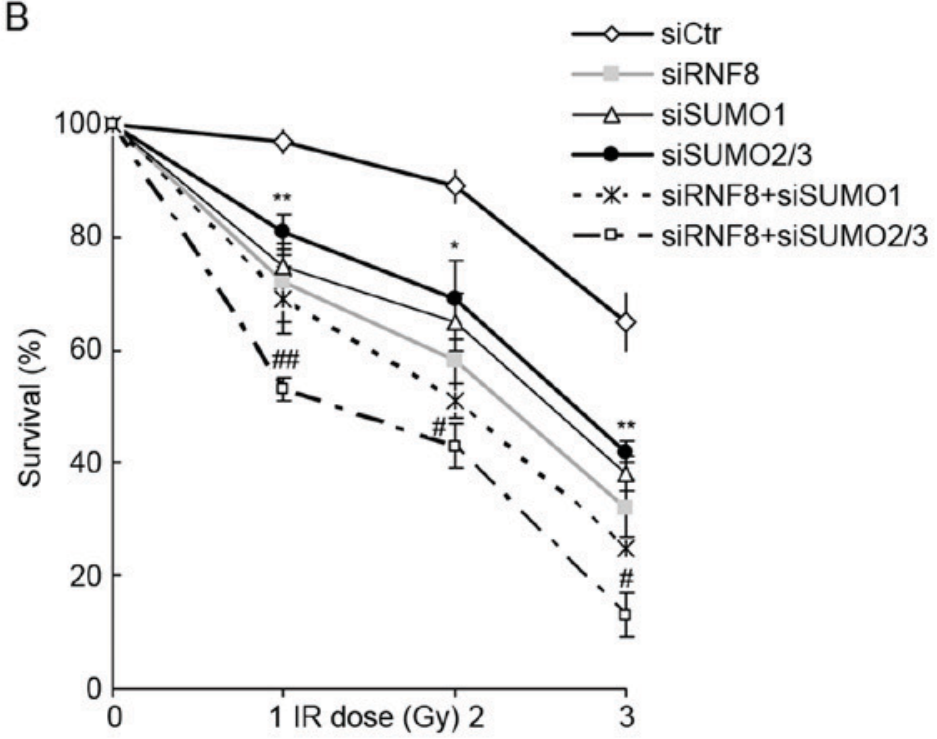

Figure 3. RNF8 and SUMO2/3 are localized to sites of DNA lesions and required for cell survival following DNA damage. (A) Co-localization of RNF8 and SUMO2/3 at sites of DNA lesions in U2OS cells. U2OS cells were transfected with Flag-RNF8 or co-transfected with Flag-RNF8 and HA-SUMO2 for 28 h. The cells were irradiated with $10 \mathrm{~Gy}$ or left untreated and immunostained with anti-Flag, anti- $\gamma$-H2AX and anti-HA antibodies. The cells were observed under a Leica confocal microscope. Magnification, x630. (B) Survival of siRNA-transfected U2OS cells exposed to irradiation. U2OS cells were transfected with siRNAs for $48 \mathrm{~h}$, followed by exposure to different doses of irradiation and left to grow for 10 days. The cells were then fixed and stained with crystal violet, and the number of colonies was counted. The experiment was performed in triplicate and data are presented as the mean \pm standard deviation of three independent experiments. ${ }^{* *} \mathrm{P}<0.01$ and ${ }^{*} \mathrm{P}<0.05$, vs. siCtr group; ${ }^{\# \#} \mathrm{P}<0.01$ and ${ }^{\#} \mathrm{P}<0.05$, vs. siRNF8 group. RNF8, ring finger protein 8; SUMO, small ubiquitin-like modifier; si/siRNA, small interfering RNA; Ctr, control; HA, hemaglutinin; IR irradiation. 
beyond the effect of either RNF8 or SUMO2/3 alone. However, this was not observed in the cells co-transfected with siRNAs targeting RNF8 and SUMO1. Together, these results suggested that binding of RNF8 to SUMO2/3 promoted DNA repair.

\section{Discussion}

Although the sumoylation pathway has been well characterized to have a significant role in the mammalian DNA damage response, the functional properties of SUMO paralogues, which mediate noncovalent binding to targeting proteins in the cellular response to genotoxic stress, remain to be fully elucidated. In the present study, a direct, noncovalent interaction was identified between RNF8 and SUMO2/3. RNF8 and SUMO2/3 co-localize at the sites of DNA damage following genotoxic stress. The direct binding of RNF8 to SUMO2/3 is required for cell survival upon DNA damage. The results of the present study suggested that RNF8 mediated the cooperation between the ubiquitin and SUMO systems in the cellular response to DNA damage.

Sumoylation has been well characterized as a pervasive mechanism for controlling the cellular response to DSBs. A direct link between sumoylation and DSB responses in mammalian cells was identified through observation that SUMO1, SUMO2/3 and the E3 ligase enzymes, PIAS4 and PIAS1, accumulated at DSB sites (10). In addition, in the absence of PIAS proteins, DNA damage response factors, including RNF168, 53BP1, BRCA1 and RAP80, do not co-localize to the DSB, and DNA repair is impeded $(10,11)$. In this context, SUMO often provides a binding platform for attracting coregulatory proteins, which contain a SIM to a sumoylated protein. The SIM-mediated protein-protein interaction drives key processes in the DNA damage repair response by the regulation of ubiquitin ligase activity and the promotion of SUMO-targeted E3 ubiquitin ligase (STUbL)-dependent ubiquitination of SUMO-conjugated target protein. The sumoylation of BRCA1 increases the activity of BRCA1 ubiquitin ligase, possibly via a productive association with SIM-containing target proteins (11). The STUbL RNF4 is recruited to DNA lesions via binding of its $\mathrm{N}$-terminal SIMs to sumoylated DNA-damage response proteins, including 53BP1, MDC1 and replication protein A, where it mediates the accumulation of ubiquitin adducts, which may be coupled with target degradation $(13,15)$. In the present study, data revealed that the E3 ubiquitin ligase, RNF8, bound directly to SUMO2/3 and that this interaction was important for DSB repair. Whether the binding of RNF8 to SUMO2/3 alters its ubiquitin ligase activity and whether RNF8 is an STUbL remains to be elucidated.

SIM is characterized by a loose consensus sequence with multiple variants. The first SIM was identified as a common Ser-X-Ser sequence surrounded by a hydrophobic core and acidic amino acids (16), whereas a study by Song et al suggested that SIM was the hydrophobic core with the consensus Val/Ile-X-Val/Ile-Val/Ile (17). Subsequently, Hannich et al defined SIM as a hydrophobic core flanked by acidic residues (18). It has also been suggested that hydrophobic and acidic residues in SIMs may determine its specificity in binding to distinct SUMO isoforms (19). Hecker et al showed that the presence of acidic amino acids or phosphorylated residues are necessary for the binding to SUMO1, but not to SUMO2/3 (12). Certain SUMO-binding partners do not contain one of the universal SIMs, including the I-V-D-V SIM motif in TTRAP and V-Q-E-V in thymine DNA glycosylase $(12,20)$. In the present study, it was found that the FHA domain of RNF8 was responsible for the binding to SUMO2/3. The FHA domain does not contain the universal SIM motif, and characterization of the residues in the FHA domain responsible for the binding to SUMO2/3 is required.

In conclusion, the present study described the noncovalent interaction between the E3 ubiquitin ligase RNF8 and SUMO2/3 and indicated that this interaction promoted DSB repair.

\section{Acknowledgements}

The present study was supported by the China Postdoctoral Science Foundation (grant no. 2015T80145).

\section{References}

1. Jackson SP and Bartek J: The DNA-damage response in human biology and disease. Nature 461: 1071-1078, 2009.

2. Park JM, Choi JY, Yi JM, Chung JW, Leem SH, Koh SS and Kang TH: NDR1 modulates the UV-induced DNA-damage checkpoint and nucleotide excision repair. Biochem Biophys Res Commun 461: 543-548, 2015.

3. Jackson SP and Durocher D: Regulation of DNA damage responses by ubiquitin and SUMO. Mol Cell 49: 795-807, 2013.

4. Mailand N, Bekker-Jensen S, Faustrup H, Melander F, Bartek J, Lukas C and Lukas J: RNF8 ubiquitylates histones at DNA double-strand breaks and promotes assembly of repair proteins. Cell 131: 887-900, 2007.

5. Huen MS, Grant R, Manke I, Minn K, Yu X, Yaffe MB and Chen J: RNF8 transduces the DNA-damage signal via histone ubiquitylation and checkpoint protein assembly. Cell 131: 901-914, 2007.

6. Stucki M, Clapperton JA, Mohammad D, Yaffe MB, Smerdon SJ and Jackson SP: MDC1 directly binds phosphorylated histone $\mathrm{H} 2 \mathrm{AX}$ to regulate cellular responses to DNA double-strand breaks. Cell 123: 1213-1226, 2005.

7. Yoshioka T, Kimura M, Saio M, Era S and Okano Y: Plk1 is negatively regulated by RNF8. Biochem Biophys Res Commun 410: 57-61, 2011.

8. Xu G, Chapman JR, Brandsma I, Yuan J, Mistrik M, Bouwman P, Bartkova J, Gogola E, Warmerdam D, Barazas M, et al: REV7 counteracts DNA double-strand break resection and affects PARP inhibition. Nature 521: 541-544, 2015.

9. Mattiroli F, Vissers JH, van Dijk WJ, Ikpa P, Citterio E, Vermeulen W, Marteijn JA and Sixma TK: RNF168 ubiquitinates $\mathrm{K} 13-15$ on $\mathrm{H} 2 \mathrm{~A} / \mathrm{H} 2 \mathrm{AX}$ to drive DNA damage signaling. Cell 150: 1182-1195, 2012.

10. Galanty Y, Belotserkovskaya R, Coates J, Polo S, Miller KM and Jackson SP: Mammalian SUMO E3-ligases PIAS1 and PIAS4 promote responses to DNA double-strand breaks. Nature 462: 935-939, 2009.

11. Morris JR, Boutell C, Keppler M, Densham R, Weekes D, Alamshah A, Butler L, Galanty Y, Pangon L, Kiuchi T, et al: The SUMO modification pathway is involved in the BRCA1 response to genotoxic stress. Nature 462: 886-890, 2009.

12. Hecker CM, Rabiller M, Haglund K, Bayer P and Dikic I: Specification of SUMO1- and SUMO2-interacting motifs. J Biol Chem 281: 16117-16127, 2006.

13. Yin Y, Seifert A, Chua JS, Maure JF, Golebiowski F and Hay RT: SUMO-targeted ubiquitin E3 ligase RNF4 is required for the response of human cells to DNA damage. Genes Dev 26: 1196-1208, 2012.

14. Kim ET, Kim KK, Matunis MJ and Ahn JH: Enhanced SUMOylation of proteins containing a SUMO-interacting motif by SUMO-Ubc9 fusion. Biochem Biophys Res Commun 388: 41-45, 2009. 
15. Galanty Y, Belotserkovskaya R, Coates J and Jackson SP: RNF4, a SUMO-targeted ubiquitin E3 ligase, promotes DNA double-strand break repair. Genes Dev 26: 1179-1195, 2012.

16. Minty A, Dumont X, Kaghad M and Caput D: Covalent modification of p73alpha by SUMO-1. Two-hybrid screening with p73 identifies novel SUMO-1-interacting proteins and a SUMO-1 interaction motif. J Biol Chem 275: 36316-36323, 2000.

17. Song J, Durrin LK, Wilkinson TA, Krontiris TG and Chen Y: Identification of a SUMO-binding motif that recognizes SUMO-modified proteins. Proc Natl Acad Sci USA 101: $14373-14378,2004$
18. Hannich JT, Lewis A, Kroetz MB, Li SJ, Heide H, Emili A and Hochstrasser M: Defining the SUMO-modified proteome by multiple approaches in Saccharomyces cerevisiae. J Biol Chem 280: 4102-4110, 2005.

19. Kerscher O: SUMO junction-what's your function? New insights through SUMO-interacting motifs. EMBO Rep 8: 550-555, 2007.

20. Baba D, Maita N, Jee JG, Uchimura Y, Saitoh H, Sugasawa K, Hanaoka F, Tochio H, Hiroaki H and Shirakawa M: Crystal structure of thymine DNA glycosylase conjugated to SUMO-1. Nature 435: 979-982, 2005. 